\title{
Exactitud y utilidad diagnóstica de la IgM en infecciones por Bartonella henselae
}

\author{
Katia Abarca, Matías Winter, Delphine Marsac, Carlos Palma, Ana M. Contreras y Marcela Ferrés
}

\section{Accuracy and diagnostic utility of $\operatorname{IgM}$ in Bartonella henselae infections}

Introduction: Laboratory diagnosis of cat scratch disease (CSD) is based on the determination of specific antibodies anti-Bartonella henselae by different techniques. The CDC recommends IgG by immunofluorescent assay (IFA) as the gold standard. Objective: To determine the accuracy and diagnostic utility of anti-B.henselae IgM by IFA for CSD. Material and Methods: Anti-B. henselae IgG was determined in serum of 108 patients with CSD suspicion; in addition, specific IgM was determined separately and blindly by two thoroughly trained laboratory professionals. We calculated sensitivity (S), specificity (Sp), predictive values both positive (PPV) and negative (NPV), and likelihood ratio (LR) for IgM positive (LR +) and negative (LR-). Results: In 37 patients with positive anti-B.henselae $\operatorname{IgG}$, IgM was positive in 16 and negative in 21 ; in 71 patients with negative $\operatorname{IgG}$, $\operatorname{IgM}$ was negative in 69 and positive in 2. Therefore, IgM showed S 43\%, E 97\%, PPV 88\%, NPV 77\%, LR (+) 15 and LR (-) 0.58. Conclusions: The results show that a positive IgM supports, but a negative one does not rule out a $B$. henselae infection. Therefore, IgG should be still considered as the gold standard for the diagnosis of CSD.

Key words: Bartonella henselae, cat scratch disease, IgM, IgG.

Palabras clave: Bartonella henselae, enfermedad por arañazo de gato, IgM, IgG.

\section{Introducción}

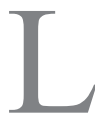

a enfermedad por arañazo de gato (EAG) causada por Bartonella henselae suele manifestarse como un cuadro de adenopatías regionales de evolución subaguda, el que puede acompañarse de otros síntomas como fiebre y compromiso del estado general, habitualmente leves. En un porcentaje variable de los casos, se presenta con manifestaciones atípicas, las que incluyen síndrome febril sin foco, granulomas hepato-esplénicos, neuroretinitis, convulsiones, osteomielitis, entre otras. En sujetos inmunocomprometidos puede presentarse como endocarditis, bacteriemia, angiomatosis bacilar y peliosis hepática ${ }^{1,2}$.

El reservorio de $B$. henselae es el gato doméstico, cuyo nivel de infección estimado por estudios de seroprevalencia fluctúa entre 15-44\% en Estados Unidos de América (E.U.A.), 47,5\% en Singapur y $36 \%$ en Francia ${ }^{3,4}$. En Chile, se ha reportado una seroprevalencia global en gatos de tres regiones del país, de $85,6 \%$, con $41,7 \%$ de animales bacteriémicos ${ }^{5}$.

La prevalencia de la infección en humanos es variable en distintos estudios. En niños se ha encontrado seropositividad de 15 y 18,5\% en Grecia y Canadá, respectivamente $^{6,7}$. En adultos, las cifras varían entre 3 y $6 \%$ en E.U.A. continental, Hawai, España y Japón ${ }^{8-10}$ hasta $37 \%$ en Canadá ${ }^{11}$. En nuestro país se ha reportado una prevalencia de $13,3 \%$ en niños y de $10,3 \%$ en adultos con riesgo ocupacional ${ }^{12}$.

La enfermedad clínica y sus manifestaciones atípicas son de creciente reconocimiento en nuestro país ${ }^{13-16}$, constituyendo un diagnóstico frecuente en la práctica clínica pediátrica. Por ello es relevante contar con técnicas de laboratorio confiables para el médico clínico, en particular para el diagnóstico diferencial de la presentación atípica de la enfermedad o cuando el antecedente epidemiológico del contacto con gatos no es evidente.

El diagnóstico de laboratorio de la EAG suele basarse en la detección de anticuerpos séricos específicos; más recientemente se ha incorporado la detección de material genético de la bacteria mediante RPC, cuando se dispone de una muestra del tejido afectado. El cultivo a partir de muestras clínicas es extremadamente complejo y de bajo rendimiento, por lo que no se utiliza en la práctica ${ }^{17}$. A pesar de no otorgar un diagnóstico de certeza pues no identifica directamente al agente, el método más utilizado es la detección de anticuerpos específicos anti- $B$. henselae, ya sea de tipo IgG o IgM, por técnicas de inmunofluorescencia (IF) o inmunoensayo enzimático (ELISA). Los CDC (Centers for Disease Control and Prevention de E.U.A.) recomiendan como estándar de oro la IgG mediante IF, para la cual se reporta una sensibilidad de $88-100 \%$ y una especificidad de $92-98 \%{ }^{18}$. El título de corte recomendado por el CDC para el diagnóstico de infección es de 1/64; en Chile, después de una evaluación de prevalencia de IgG anti $B$. henselae se estableció un
Pontificia Universidad Católica de Chile, Santiago. Escuela de Medicina División de Pediatría (KA, MW, MF) Laboratorio de Infectología y Virología Molecular (DM, CP; AMC)

Los autores declaran no tener conflicto de interés.

Financiamiento: Laboratorio de Infectología y Virología Molecular, Pontificia Universidad Católica de Chile.

Recibido: 11 de julio de 2012 Aceptado: 5 de enero de 2013

Correspondencia a: Katia Abarca Villaseca katia@med.puc.cl 
título de corte en dilución de $1 / 256$, debido a que más del $10 \%$ de los sujetos estudiados, niños y personas con riesgo ocupacional como veterinarios, tenían títulos de 1/6412.

En los últimos años se han desarrollado diversos ensayos para la detección de $\operatorname{IgM}$ anti- $B$. henselae. Su sensibilidad varía en distintos estudios entre $71,4 \%$ y $95 \% \%^{1,19}$, siendo ésta menor con las técnicas de ELISA comparada con IF $(73 \% \text { contra } 93 \%)^{20}$. Con relación a especificidad, los ensayos que determinan IgM e IgG anti$B$. henselae han mostrado baja o nula reactividad cruzada con Epstein Barr, CMV, Toxoplasma gondii y Chlamydophila pneumoniae ${ }^{21,22}$, y significativa reactividad cruzada con Coxiella burnetti y Bartonella quintana ${ }^{23}$.

Si bien la sensibilidad y especificidad son las propiedades más difundidas y utilizadas de los test diagnósticos, el mejor indicador para el médico tratante es la razón de probabilidades o likehood ratio (LR). Este permite estimar la probabilidad que un paciente efectivamente tenga la enfermedad si presenta un resultado positivo $(\mathrm{LR}+)$ y la probabilidad que no la tenga si presenta un resultado negativo (LR-). Las diferencias entre las probabilidades pre y post-test pueden mover al médico clínico fuera del área de incertidumbre, ya sea apoyando o descartando su hipótesis diagnóstica clínica ${ }^{24}$.

El objetivo primario de este estudio es determinar la exactitud diagnóstica de la IgM comparada con la IgG para el diagnóstico de infecciones por $B$. henselae en pacientes con sospecha de la enfermedad.

\section{Pacientes y Métodos}

Se estudiaron 108 pacientes chilenos en quienes el médico tratante, en su mayoría infectólogos, solicitara determinación de IgG anti- $B$. henselae por sospecha clínica de EAG, al Laboratorio de Infectología y Virología Molecular de la Pontificia Universidad Católica de Chile, entre enero de 2010 y mayo de 2011.

Se realizó un estudio de potencia para el número de muestras obtenidas en ese período $(\mathrm{n}=108)$, considerando una prevalencia de 34\% y una significancia del $99 \%$, obteniendo una potencia de la muestra de $95 \%$.

Los sueros fueron procesados para la detección de

Tabla 1. Tabla de contingencia 2 × 2 para los cálculos de las propiedades de la lgM anti-Bartonella henselae

\begin{tabular}{|llll|} 
& $\begin{array}{l}\text { Enfermo } \\
\text { (IgG positiva) }\end{array}$ & $\begin{array}{l}\text { Sano } \\
\text { (IgG negativa) }\end{array}$ & Total \\
IgM positiva & 16 & 2 & 18 \\
IgM negativa & 21 & 69 & 90 \\
Total & 37 & 71 & 108 \\
\hline
\end{tabular}

S 43\%, E $97 \%$, VPP $88 \%$, VPN 77\%, LR(+) 15,3 y LR(-) 0,58.
IgG anti- $B$. henselae en la dilución habitual de 1/256 y almacenados a $-20^{\circ} \mathrm{C}$ hasta el procesamiento de la $\operatorname{IgM}$, la cual fue realizada en dilución de $1 / 20$, según recomienda el fabricante. $\operatorname{La} \operatorname{IgM}$ fue realizada entre 0 y 108 días después que la IgG (mediana 18 días) y su lectura fue realizada por separado por dos profesionales vastamente entrenados en el diagnóstico serológico de $B$. henselae, que no conocieron los antecedentes clínicos de los pacientes ni los resultados de las lecturas realizadas por su par. Ambas determinaciones se realizaron mediante IF, utilizando kits comerciales (Focus ${ }^{\circledR}$, USA), siguiendo las instrucciones del fabricante.

En tres pacientes que tenían más de una muestra, se utilizó sólo la primera de ellas.

El estudio fue aprobado por el Comité de Ética de la Dirección de Investigación de la Escuela de Medicina de la Pontificia Universidad Católica. Por su carácter retrospectivo y la confidencialidad de los datos que identifican al paciente, el comité de ética otorgó una dispensa al Consentimiento Informado.

Se calculó sensibilidad (S), especificidad (E), valores predictor positivo (VPP), negativo (VPN) y likelihood ratio (LR) para una IgM positiva (LR+) y negativa (LR-). Los cálculos fueron realizados utilizando el programa SPSS 17.0. Las probabilidades post-test se determinaron mediante el nomograma de Fagan uniendo con una línea la probabilidad pre-test (prevalencia de la enfermedad en esta serie clínica) y los LR calculados.

\section{Resultados}

De los 108 pacientes analizados, en 37 de ellos la IgG anti- $B$. henselae resultó positiva. De ellos, la IgM fue positiva en 16 y negativa en 21 . De los 71 pacientes con IgG negativa, $\operatorname{la} \operatorname{IgM}$ fue negativa en 69 y positiva en 2 . Por consiguiente, la IgM resultó tener una S 43\%, E 97\%, VPP $88 \%$, VPN 77\%, LR(+) 15,3 y LR(-) 0,58 (Tabla 1). La Figura 1 muestra las probabilidades post-test utilizando los LR en el nomograma de Fagan. La Figura 2 muestra un suero positivo para IgG en dilución 1/256.

\section{Discusión}

Para el diagnóstico de EAG, la IgM anti-B. henselae por IF demostró alta E y VPP pero baja S y VPN. Un estudio holandés mostró resultados similares, con una especificidad de $93 \%$ y una sensibilidad de $53 \%{ }^{25}$.

La IgM mostró un LR positivo elevado, lo que sumado a la probabilidad pre-test de 34,3\% (prevalencia de EAG en esta muestra), indica que un resultado positivo del test aumenta la probabilidad diagnóstica a 89\% (IC95\% 66-97). En cambio, un resultado negativo reduce la probabilidad diagnóstica a 23\% (IC95\% 19-29). Esta 


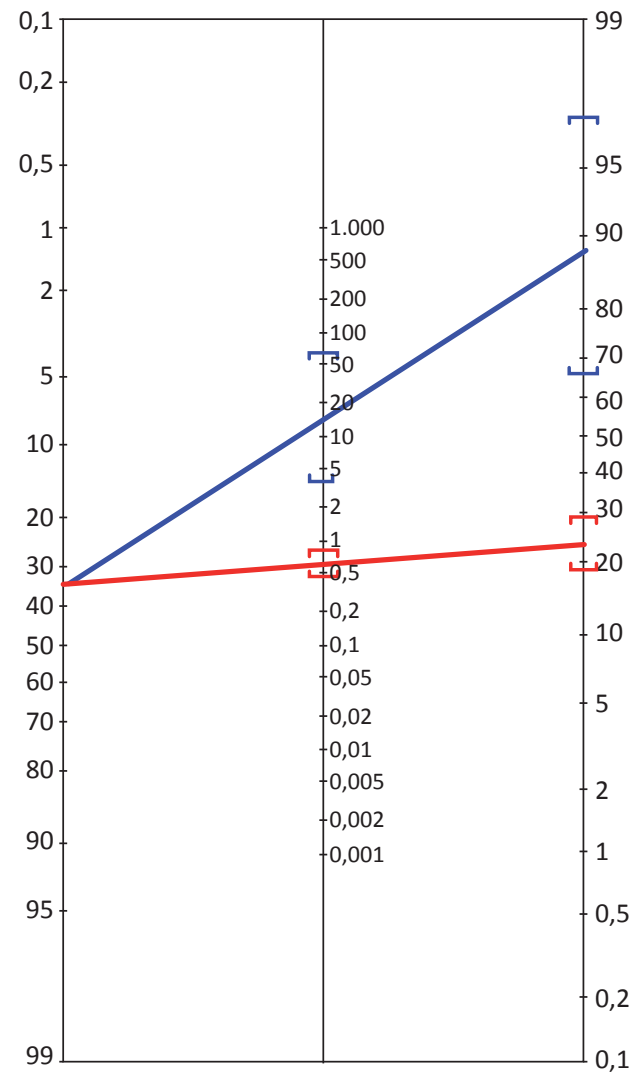

Prob. pre-test Likelihood ratio Prob. post-test

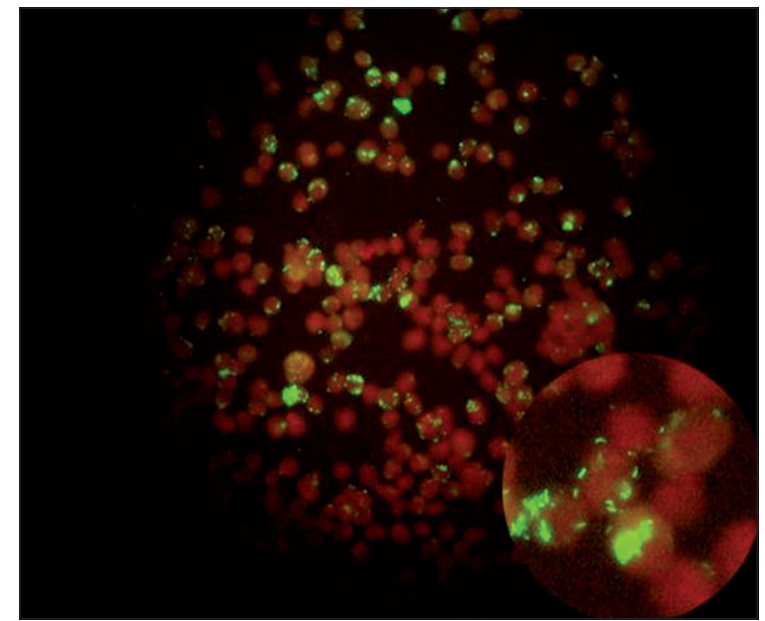

Figura 2. Imagen que muestra un suero con presencia de anticuerpos lgG anti-Bartonella henselae mediante inmunofluorescencia indirecta (IFI) en dilución 1/256. tuvieron una IgG negativa luego seroconvertieron ${ }^{26}$. Por su parte la IgM suele negativizarse a los 3 meses de iniciados los síntomas en la mayoría de los pacientes, lo que debe ser considerado al estudiar a pacientes con síntomas prolongados ${ }^{26}$.

Algunas limitaciones propias de nuestro estudio son su carácter retrospectivo, y el haber trabajado sólo con una muestra por paciente, ya que ante resultados cercanos al punto de corte una segunda muestra puede definir mejor el estado serológico del sujeto.

A pesar de estas limitaciones, nuestros datos muestran que la IgM no cumple aún los requisitos para reemplazar a la IgG en el diagnóstico de infecciones por $B$. henselae; por tanto, esta última debe seguir considerándose como el estándar de oro, mientras no se disponga de exámenes de certeza que identifiquen en forma directa el agente, ya sea mediante técnicas moleculares o de aislamiento.

Agradecimientos. Al personal del Laboratorio de Infectología y Virología Molecular de la Pontificia Universidad Católica de Chile.

\section{Resumen} que un resultado de IgM positivo podría tener utilidad en apoyar el diagnóstico de EAG, pero uno negativo no permite descartarlo. Por lo tanto, la IgM no agrega mayor beneficio con relación a la IgG en el diagnóstico de EAG, y en caso de usar ambas, se aumentarían los costos sin lograr una mayor utilidad diagnóstica.

Existen ciertas limitaciones inherentes a los test serológicos utilizados para diagnóstico de infecciones agudas. Cuando una determinación de $\operatorname{IgG}$ es realizada muy precozmente en el curso de la enfermedad, un resultado negativo con alta sospecha clínica debería motivar repetir el examen en días posteriores. Un estudio, realizado con técnica de ELISA, reportó que 27 de 90 pacientes que
Introducción: El diagnóstico de laboratorio de la enfermedad por arañazo de gato (EAG) se basa en la determinación de anticuerpos específicos anti-Bartonella henselae por distintas técnicas. El CDC de E.U.A. recomienda como estándar de oro la IgG mediante inmunofluorescencia (IF). Objetivo: Determinar la exactitud y utilidad diagnóstica de la IgM anti- $B$. henselae por IF para EAG. Material y Método: En suero de 108 pacientes a quienes se realizó IgG anti- $B$. henselae por sospecha de EAG, se determinó la presencia de IgM específica, en forma separada y ciega por dos profesionales de laboratorio ampliamente entrenados. Se calculó sensibilidad (S), 
especificidad (E), valores predictores positivo (VPP) y negativo (VPN) y likelihood ratio (LR) para una IgM positiva (LR+) y negativa (LR-). Resultados: En 37 pacientes con IgG anti- $B$. henselae positiva, la IgM fue positiva en 16 y negativa en 21; en 71 pacientes con IgG negativa, la IgM fue negativa en 69 y positiva en 2 . Por consiguiente, la IgM presentó S 43\%, E 97\%, VPP 88\%, VPN 77\%, LR(+) 15 y LR(-) 0,58. Conclusiones: Los resultados sugieren que una IgM positiva apoya el diagnóstico de EAG, pero una negativa no permite descartarlo. Por tanto, la IgG debe seguir considerándose como el estándar de oro para el diagnóstico de infecciones por $B$. henselae.

\section{Referencias bibliográficas}

1.- Bass J, Vincent J, Person D. Expanding spectrum of Bartonella infections: Cat scratch disease. Pediatric Infect Dis J 1997; 16: 163-79.

2.- Chang C, Chomel B, Kasten R, Tappero J, Sánchez M, Koehler J. Molecular epidemiology of Bartonella henselae infection in human inmunodeficiency virus-infected patients and their cat contacts, using pulsed-field gel electrophoresis and genotyping. J Infect Dis 2002; 186: 1733-9.

3.- Jameson P, Greene C, Regnery R, Dryden M, Marks A, Brown J, et al. Prevalence of Bartonella henselae antibodies in pet cats throughout regions of North America. J Infect Dis 1995; 172: 1145-9.

4.- Nasirudeen A M, Thong M L. Prevalence of Bartonella henselae immunoglobulin G antibodies in Singaporean cats. Pediatr Infect Dis J 1999; 18: 276-8.

5.- Ferrés M, Abarca K, Godoy P, et al. Presence of Bartonella henselae in cats: natural reservoir quantification and human exposition risk of this zoonoses in Chile. Rev Med Chile 2005; 133 : 1465-71.

6.- Antoniou M, Economou I, Wang X, Psaroulaki A, Spyridaki I, Papadopoulos B, et al. Fourteen-year seroepidemiological study of zoonoses in a Greek village. Am J Trop Med Hyg 2002; 66: 80-5.

7.- Cimolai N, Benoit L, Hill A, Lyons C. Bartonella henselae infection in British Columbia: evidence for an endemic disease among humans. Can J Microbiol 2000; 46: 908-12.

8.- Regnery R L, Olson J G, Perkins B A, Bibb W. Serological response to Rochalimaea henselae antigen in suspected cat-scratch disease. Lancet 1992; 339: 1443-5.
9.- Demers D M, Bass J W, Vincent J M, Person D A, Noyes D K, Staege C M, et al. Cat-scratch disease in Hawaii: etiology and seroepidemiology. J Pediatr 1995; 127: 23-6.

10.- Kikuchi E, Maruyama S, Sakai T, Tanaka S, Yamaguchi F, Hagiwara T, et al. Serological investigation of Bartonella henselae infections in clinically cat-scratch disease-suspected patients, patients with cardiovascular diseases, and healthy veterinary students in Japan. Microbiol Immunol 2002; 46: 313-6.

11.- Sander A, Posselt M, Oberle K, Bredt W. Seroprevalence of antibodies to Bartonella henselae in patients with cat scratch disease and in healthy controls: evaluation and comparison of two commercial serological tests. Clin Diagn Lab Immunol 1998; 5: 486-90.

12.- Ferrés M, Abarca K, Prado P, Montecinos L, Navarrete M, Vial P. Prevalence of Bartonella henselae antibodies in Chilean children, adolescents and veterinary workers. Rev Med Chile 2006; 134: 863-7.

13.- Abarca K, Vial P A, Rivera M, García C, Oddó D, Prado P et al. Bartonella henselae infection in immunocompetent patients: cat scratch disease. Rev Med Chile 1996; 124 : 1341-9.

14.- Täger M, Zamorano J. Osteomielitis, una manifestación inusual de la enfermedad por arañazo de gato. Rev Chilena Infectol 2000; 17: 326-31.

15.- Navarrete M, Täger M, Wenzel S, Podestá L, Pincheira M, Zaror L. Formas atípicas de enfermedad por arañazo de gato. Rev Chilena Infectol 2003; 20: 257-61.

16.- Wolff E, Muñoz M P, Zapata C, Ledermann W. Enfermedad por arañazo de gato complicada con compromiso sistémico, osteomielitis vertebral y absceso paravertebral. Rev Chilena Infectol 2000; 17: 332-9.

17.- La Scola B, Raoult D. Culture of Bartonella quintana and Bartonella henselae from human samples: a 5-year experience. J Clin Microbiol 1999; 1899-905.

18.- English R. Cat-scratch disease. Pediatr Rev 2006; 123-8.

19.- Bergmans A, Peeters M, Schellekens J, Vos M, Sabbe L, Ossewaarde J et al. Pitfalls and fallacies of cat scratch disease serology: evaluation of Bartonella henselae-based indirect fluorescence assay and enzyme-linked immunoassay. J Clin Microbiol 1997; 35: 1931-7.

20.- Szelc-Kelly C, Goral S, Pérez-Pérez G, Perkins B, Regnery R, Edwards K. Serologic responses to Bartonella and Afipia antigens in patients with cat scratch disease. Pediatrics 1995; 96: 1137-42.

21.- Sander A, Berner R, Ruess M. Serodiagnosis of cat scratch disease: response to Bartonella henselae in children and a review of diagnostic methods. Eur J Clin Microbiol Infect Dis 2001; 20: 392-401.

22.- Giladi M, Kletter Y, Avidor B, MetzkorCotter E, Varon M, Golan Y, et al. Enzyme immunoassay for the diagnosis of cat-scratch disease defined by polymerase chain reaction. Clin Infect Dis 2001; 33: 1852-8.

23.- La Scola B, Raoult D. Serological crossreactions between Bartonella quintana, Bartonella henselae, and Coxiella burnetii. J Clin Microbiol 1996; 34: 2270-4.

24.- Valenzuela L, Cifuentes L. Validity of diagnostic tests. Rev Med Chile 2008; 136: 401-4.

25.- Vermeulen M J, Verbakel H. Evaluation of sensitivity, specificity and cross-reactivity in Bartonella henselae serology. J Med Microbiol 2010; 59: 743-5.

26.- Metzkor-Cotter E, Kletter Y, Avidor B. Longterm serological analysis and clinical follow-up of patients with cat scratch disease. Clin Infect Dis 2003; 37: 1149-54. 